Article original

\title{
L'adhésion des urologues maghrébins au programme de réhabilitation améliorée après cystectomie radicale
}

\author{
The adherence of Maghreb urologists to the rehabilitation program improved \\ after radical cystectomy \\ Slimane KERROUMI 1,2, Salim TALEB ${ }^{1,2}$, Chihab OUANEZAR ${ }^{1,2}$, Adil Djilali LANSARI ${ }^{1,2}$, Ali BAZZI ${ }^{1,2}$, \\ Mohamed Amine CHELEF', Malik HAFFAF', Mustefa Jamal YOUSFI',2 \\ 'Service d'Urologie, Etablissement Hospitalier et Universitaire d'Oran. \\ ¿Laboratoire de recherche Santé et Environnement. Faculté de Médecine d’Oran
}

Auteur correspondant: kerroumi.slimane@hotmail.fr

Article reçu le 1/09/2018 Accepté le 26/06/2019

\section{MOTS CLÉS}

Société de réhabilitation améliorée après chirurgie; Fast-track; cancer de vessie; Cystectomie

\section{Résumé}

L'objectif de cette enquête est d'évaluer l'application du programme de réhabilitation améliorée après une cystectomie totale Par les urologues maghrébins. L'enquête a été réalisée en 2017 auprès des urologues maghrébins affiliés à leurs sociétés savantes respectives, la société marocaine d'urologie (AMU), la société tunisienne d'Urologie (STU), la société algérienne de chirurgie urologique (SACU) et l'association algérienne d'urologie (AAU). L'auto-questionnaire a été élaboré sur les items du programme de la société de réhabilitation améliorée après chirurgie (ERAS). Il a été donné aux urologues pendant leurs congrès respectifs.

Le nombre de questionnaires remplis est de 221 sur 383 distribués dont 115 (52,03\%) ont été remplis par des urologues algériens, 85 (38,5\%) par des urologues marocains et $21(9,5 \%)$ par les urologues tunisiens. Les médecins hospitalo-universitaires, les hospitaliers et les privés représentent respectivement $43 \%, 21,3 \%$ et $35,7 \%$. Les urologues déclarent qu'ils ont un programme de prévention des accidents thromboemboliques, l'hypothermie et une analgésie péridurale pour diminuer la douleur post-opératoire dans $69,6 \%$. 75,6\% respectent les recommandations de la réhabilitation postopératoire avec un taux de $64.7 \%$ du lever précoce, la prévention des nausées et vomissements dans $82,8 \%$, l'absence d'utilisation des morphiniques est estimée à $80,1 \%$. La reprise de l'alimentation en post-opératoire est appliquée dans $39,4 \%$ des cas. L'état nutritionnel en préopératoire, la préparation digestive avec un régime sans résidu diminue la durée d'hospitalisation ( $p=0,032, p=0.01, p=0,009)$.

Cette enquête montre que plus de la moitié des urologues maghrébins interrogés déclarent qu'ils suivent les recommandations du programme de réhabilitation améliorée afin de diminuer la morbidité et de réduire la durée d'hospitalisation, bien que l'autorisation de l'alimentation postopératoire précoce reste très peu suivie. 


\section{KEY WORDS}

ERAS; Fast-track; Blad-
der cancer; Cystec-
tomy

\section{Introduction}

D Le geste chirurgical est source d'un « stress " responsable de modifications hormonales, métaboliques et physiologiques qui retentissent sur la convalescence et les suites postopératoires. L'approche «Enhanced recovery after surgery» $(\operatorname{Eras} \otimes)$ - dite réhabilitation rapide après chirurgie (RRAC) ou réhabilitation améliorée (RAAC) dans les pays francophones a été introduite pour réduire le stress métabolique chirurgical [1].

La réhabilitation améliorée postopératoire se définit comme un ensemble de techniques et de méthodes qui ont pour objectif de diminuer le stress métabolique [2]. Elle est basée sur plusieurs paramètres : recentrage sur le patient, chirurgie mini-invasive et une prise en charge multi-professionnelle [3].

En chirurgie colorectale, le programme RAAC réduit considérablement les complications post-opératoires et le séjour hospitalier à travers des études de haut niveau de preuve [4]. Le programme de réhabilitation a été adopté en urologie pour la prise en charge des cystectomies depuis les recommandations ERAS 2013. Malgré la standardisation de la technique chirurgicale et l'amélioration des protocoles d'anesthésie et de soins périopératoires, la morbidité après cystectomie radicale peut atteindre jusqu'à 30-64\% [5]. Actuellement, pour qu'un service soit certifié RAAC en urologie, il doit être labellisé par une société savante de réhabilitation améliorée. Au Maghreb, aucune institution n'est certifiée RAAC en urologie. Pour cette raison, il est nécessaire d'évaluer les connaissances des urologues maghrébins et leurs pratiques en matière d'application des recommandations de la société ERAS pour la cystectomie.

\section{Méthodes}

C'est une enquête descriptive, réalisée en 2017 auprès des urologues maghrébins à travers leurs sociétés savantes respectives : la société marocaine d'urologie (AMU), la société tunisienne d'Urologie (STU), la société algérienne de chirurgie urologique (SACU) et l'association algérienne d'urologie (AAU). La population cible est définie par l'ensemble des urologues maghrébins qui pratiquent la cystectomie au sein d'une équipe dans un $\mathrm{CHU}$, dans un centre hospitalier ou dans le secteur privé. 
Le questionnaire a été établi par une équipe pluridisciplinaire qui pratique la RAAC post-cystectomie du service d'urologie à l'EHU Oran (urologue, médecin réanimateur, et nutritionniste). L'auto-questionnaire a été soumis aux urologues pendant les congrès annuels des différentes sociétés savantes en séance plénière. Il comprenait 25 questions fermées avec des réponses qui se présentaient soit sous forme dichotomique soit selon 3 propositions. Dans les réponses, il existe les recommandations de l'ERAS et d'autres pratiques reconnues communément par les urologues maghrébins. L'auto-questionnaire touche les 21 variables du programme de réhabilitation de la société ERAS extrapolé de celui de la chirurgie colorectale. Chacune ou deux questions représentent une recommandation du programme ERAS.

Trois groupes d'items contenant 7 variables : la phase préopératoire, la phase peropératoire et post-opératoire [annexe 1,2].

Les paramètres de la phase préopératoire sont : Education et information du patient, optimisation des dysfonctions d'organes (arrêt du tabac), préparation mécanique intestinale, boisson carbohydrates, médicaments sédatifs pré-anesthésiques, prophylaxie trombo-embolique, le jeûne préopératoire, état nutritionnel du patient.

Les paramètres de la phase peropératoire sont : l'analgésie épidurale, l'approche mini-invasive, le drainage du site opératoire, la prophylaxie antimicrobienne, la gestion des fluides, la prévention de l'hypothermie.

Les paramètres de la phase postopératoire sont : l'intubation naso-gastrique, la prévention de l'iléus, la prévention des nausées et vomissements, l'analgésie postopératoire, la mobilisation précoce et l'alimentation orale précoce.

L'analyse statistique a été réalisée avec le logiciel SPSS version 20 , en prenant un risque a à $5 \%$. Le test Khi 2 avec correction de Yates a était utilisé pour les caractères qualitatifs.

\section{Résultats}

Le nombre de questionnaires retournés et analysés est de 221 sur 383 distribués dont 115 (52,03\%) remplis par des urologues algériens, 85 (38,5\%) par des urologues marocains et 21 (9.5\%) par les urologues tunisiens. Les médecins hospitalo-universitaires, les hospitaliers et les privés représentent respectivement $43 \%, 21,3 \%$ et $35,7 \%$.

La plupart des centres au Maghreb pratiquent la dérivation trans-iléale type Bricker $(70,8 \%)$, le remplacement vésical dans $8,2 \%$ et l'urétérostomie cutanée bilatérale dans $21 \%$ des cas. Le remplacement vésical est représenté par la néovessie en $Z$, le remplacement camey2, le Studer et autre remplacement dans $72,4 \%, 11,3 \%, 10,9 \%$ et $5,4 \%$ respectivement. Les urologues maghrébins qui pratiquent moins de 3 cystectomies par mois représentent $78,6 \%$ et ceux qui pratiquent plus de 10 cystectomies représentent $3,2 \%$.

\section{Les paramètres préopératoires}

La plupart des urologues ne donnent pas d'importance à l'information et l'éducation du patient pour sa dérivation. 73,6\% des urologues déclarent ne jamais demander aux patients de porter une poche d'essayage et $52,7 \%$ des urologues ne font pas de marquage de poche en préopératoire. $67,9 \%$ des urologues demandent aux patients d'arrêter le tabac pendant plus de 8 semaines avant le geste opératoire mais il reste $16,3 \%$ des urologues qui ne pratiquent aucune mesure de conditionnement préopératoire. Pour la préparation digestive, 34,4\% des urologues maghrébins déclarent qu'ils conseillent à leurs patients soit aucune préparation ou un régime sans résidu pendant 5 jours avant le geste opératoire. 91,2\% des urologues insistent sur la prophylaxie thrombo-embolique en peropératoire en utilisant des moyens différents (tableau 2). L'évaluation de l'état nutritionnel intéresse la plupart des urologues (73,4\% de praticiens) avec des paramètres d'évaluation très variables entre médecins (figure 1). 50,5\% des urologues administrent de l'albumine à leurs patients d'albumine et des compléments alimentaires en cas de dénutrition.

L'analyse des courbes de ROC ont permis d'identifier que les patients avec un score TIMI $\square 4$, un Heart score $>8$ et un score de syntaxe $\square 20$ étaient également plus susceptibles d'avoir une augmentation de la mortalité à un an alors que le score de Grace n'était pas prédictif (figures 1 et 2).

Figure1. Les paramètres de l'état nutritionnel

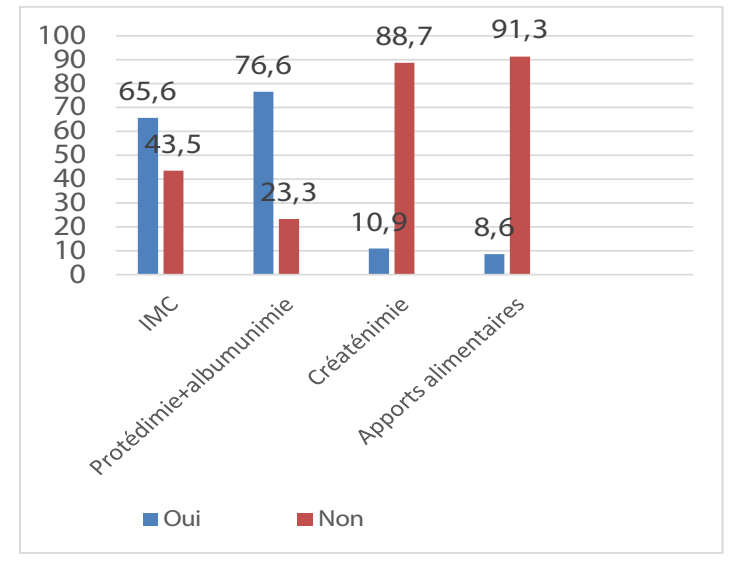


Les paramètres peropératoires

Pour la gestion des liquides, 46,8\% des urologues interrogés déclarent qu'ils sont au courant de la quantité de la flotte administrée au patient et $48,2 \%$ affirment qu'ils ne sont pas informés en peroperatoire. $81,1 \%$ des urologues interrogés pratiquent la voie d'abord ouverte pour la cystectomie, il existe $18,5 \%$ des urologues qui pratiquent la cystectomie laparoscopique. Les urologues interrogés préviennent l'hypothermie par tous les moyens disponibles dans $77 \%$ des cas.

Pour la mise en place de la sonde nasogastrique, 46,8\% des urologues la mettent systématiquement en peropératoire et $51,8 \%$ sont contre sa mise en place. La majorité des urologues sont pour l'analgésie péridurale afin d'assurer un moyen antalgique en postopératoire et de diminuer la prise des morphiniques. Pour le drainage du site opératoire, les moyens déclives sont plus utilisés que les dispositifs aspiratifs (fig 2,3 ).

\section{Figure 2. la pratique de l'analgésie péridurale}

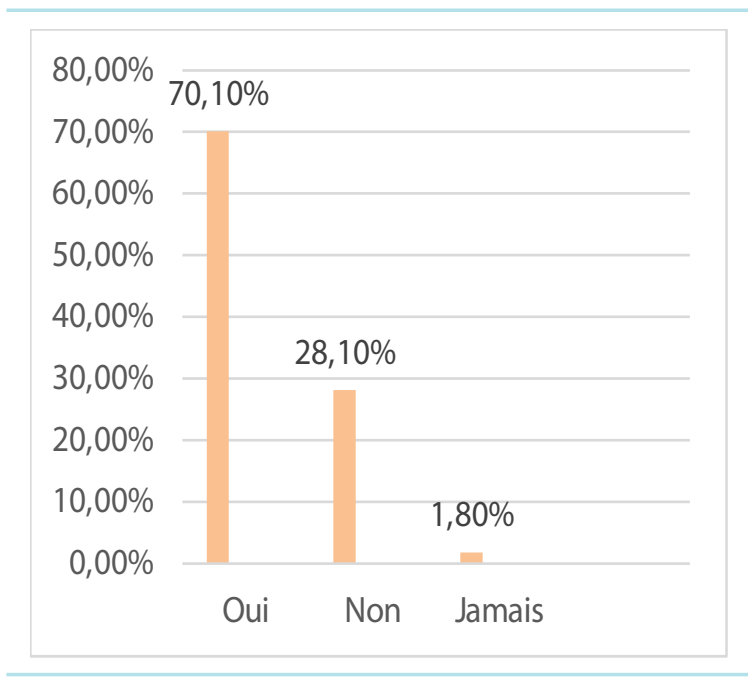

Figure 3. Le drainage du site opératoire

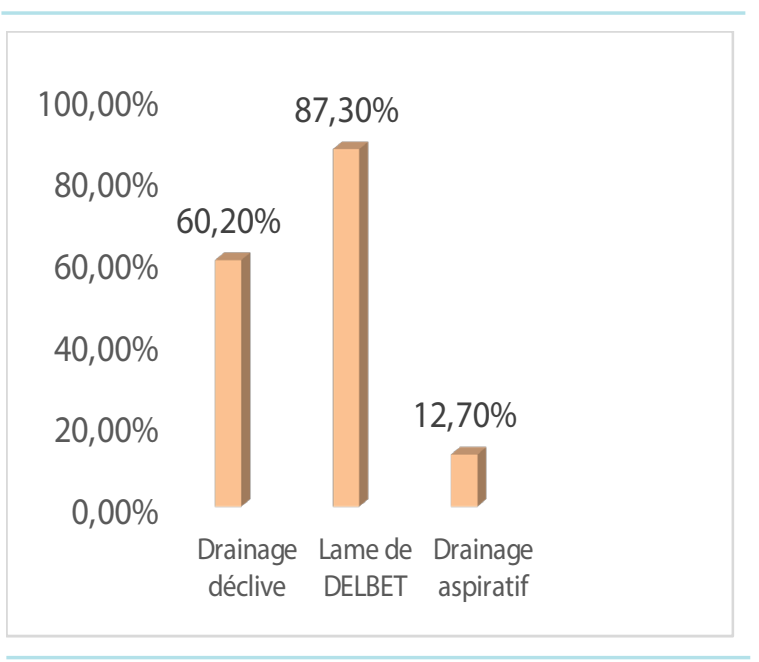

Les paramètres postopératoires

La prescription des antiémétiques en postopératoire n'est suivie que par $34,2 \%$ des équipes. $1 \%$ des médecins déclarent qu'ils n'ont jamais prescrit un antiémétique en postopératoire. Pour les paramètres du fast tract proprement dit, la plupart des médecins sont pour ne pas utiliser les morphiniques dans $80,1 \%$, le lever précoce ( $24 \mathrm{~h}$ postopératoire) dans $82,8 \%$ et retirer la sonde nasogastrique dans les $24 \mathrm{~h}$ dans $78,9 \%$. Dans le reste des items, l'avis des urologues maghrébins est exposé dans le tableau 1.

Tableau1. les paramètres pré et postopératoires

\begin{tabular}{|c|c|}
\hline & $\%$ \\
\hline \multicolumn{2}{|l|}{$\begin{array}{l}\text { Prévention d'un accident thrombo- } \\
\text { embolique }\end{array}$} \\
\hline - Héparine a bas poids moléculaire & 68,8 \\
\hline - Bas de contention & 27,4 \\
\hline $\begin{array}{l}\text { - Compression pneumatique } \\
\text { intermittente }\end{array}$ & 9,3 \\
\hline \multicolumn{2}{|l|}{ Autorisation de l'alimentation } \\
\hline - Précoce & 39,4 \\
\hline - Ablation de la SNG & 8,1 \\
\hline - $\quad$ Après reprise du transit & 52,5 \\
\hline \multicolumn{2}{|l|}{ Alimentation postopératoire } \\
\hline - Oui & 52,5 \\
\hline - Non & 31,7 \\
\hline - Jamais & 15,8 \\
\hline \multicolumn{2}{|l|}{ Mastication du chewin-gum } \\
\hline - Oui & 46,6 \\
\hline - Non & 43,4 \\
\hline - Jamais & 10 \\
\hline
\end{tabular}

Le suivi des recommandations de l'ERAS et son impact sur le séjour hospitalier

Pour le suivi des urologues des paramètres préopératoires du programme ERAS, deux variables essentielles ont été choisies, l'arrêt du tabac 8 semaines avant le geste opératoire et l'évaluation de l'état nutritionnel du patient. Le taux de suivi est de $55,7 \%$. Cependant pour les paramètres peropératoires, l'ensemble des urologues qui pratiquent une analgésie péridurale avec ceux qui utilisent tous les moyens pour prévenir l'hypothermie est de $47 \%$. On note un suivi majoritaire du programme fast track en postopératoire avec un taux de 75,6\% (la non utilisation des morphiniques, le lever précoce dans les $24 \mathrm{~h}$ et la non utilisation de l'alimentation parentérale). L'état nutritionnel en préopératoire, la préparation digestive avec un régime sans résidu diminue la durée d'hospitalisation ( $p=0,032, p=0.01, p=0,009)$ (tableau 2). 
Tableau 2. Impact des variables du programme ERAS sur la durée d'hospitalisation

\begin{tabular}{|c|c|c|c|c|c|}
\hline & \multicolumn{3}{|c|}{ Duree d'hospitalisation } & \multirow[t]{2}{*}{$p$} \\
\hline & & \multirow{2}{*}{$\begin{array}{c}\begin{array}{c}\text { Moins } \\
\text { d'une } \\
\text { semaine }\end{array} \\
29\end{array}$} & \multirow{2}{*}{$\begin{array}{c}\text { Entre 1-2 } \\
\text { semaines } \\
131,7\end{array}$} & $\begin{array}{c}\text { Plus } \\
\text { d'une } \\
\text { semaine } \\
\end{array}$ & \\
\hline \multirow{3}{*}{ Le lever précoce } & $24 \mathrm{~h}$ & & & 22,4 & \multirow{3}{*}{0,079} \\
\hline & $48 \mathrm{~h}$ & 3,6 & 16,5 & 2,8 & \\
\hline & $\begin{array}{l}\text { Libre choix } \\
\text { au patient }\end{array}$ & 2,4 & 10,8 & 1,8 & \\
\hline \multirow{3}{*}{$\begin{array}{l}\text { Morphine } \\
\text { postopératoire }\end{array}$} & Oui & 6.7 & 30.2 & 5,1 & \multirow{3}{*}{0,072} \\
\hline & Non & 28 & 127,3 & 21,6 & \\
\hline & Jamais & 0,3 & 1,4 & 0,2 & \\
\hline \multirow{3}{*}{$\begin{array}{l}\text { Nombre de } \\
\text { cystectomie }\end{array}$} & $0.3 / \mathrm{mois}$ & 27,5 & 6,7 & 0,8 & \multirow{3}{*}{0,607} \\
\hline & \begin{tabular}{|l|}
$4-10 / \mathrm{mois}$ \\
\end{tabular} & 124,2 & 30,2 & 3,6 & \\
\hline & $+10 /$ mois & 21,2 & 5,2 & 0,6 & \\
\hline \multirow{3}{*}{ Etat nutritionnel } & Oui & 25,8 & 117,3 & 19,9 & \multirow{3}{*}{0,006} \\
\hline & Non & 5,5 & 25,2 & 4,3 & \\
\hline & Parfois & 3,6 & 16,5 & 2,8 & \\
\hline \multirow{3}{*}{$\begin{array}{l}\text { Préparation } \\
\text { digestive }\end{array}$} & Non & 12 & 54.7 & 9.3 & \multirow{3}{*}{0,010} \\
\hline & $\begin{array}{l}\text { Oui avec } \\
\text { RSR }\end{array}$ & 12 & 54.7 & 9.3 & \\
\hline & $\begin{array}{l}\text { Minimum } \\
8 \mathrm{~h}\end{array}$ & 10,9 & 49,6 & 8,4 & \\
\hline \multirow{3}{*}{$\begin{array}{l}\text { Autorisation de } \\
\text { l'alimentation }\end{array}$} & Précoce & 13,8 & 62,6 & 10,6 & \multirow{3}{*}{0,522} \\
\hline & \begin{tabular}{|l} 
Après \\
ablation \\
SNG \\
\end{tabular} & 62,6 & 13 & 83,5 & \\
\hline & $\begin{array}{l}\text { Après } \\
\text { reprise du } \\
\text { transit }\end{array}$ & 10,6 & 2,2 & 14,2 & \\
\hline \multirow{3}{*}{$\begin{array}{l}\text { Mâcher du } \\
\text { chewing-gum }\end{array}$} & Oui & 16,3 & 74,1 & 12,6 & \multirow{3}{*}{0,075} \\
\hline & Non & 15,2 & 69,1 & 11,7 & \\
\hline & Jamais & 3,5 & 15,8 & 2,7 & \\
\hline
\end{tabular}

\section{Discussion}

Dans la pratique, il existe quelques centres maghrébins qui font plus de 4 cystectomies par mois $(21,4 \%)$. La plupart des urologues pratiquent la dérivation trans-iléale type Bricker $(70,8 \%)$ mais ils font moins de remplacement vésical qui est estimé à $8,2 \%$. Les données de l'étude correspondent aux données de la littérature à savoir le rapport de l'OMS en 2007 sur le recensement des dérivations à partir de plusieurs séries qui donnaient un taux de Bricker de $54 \%$ sur un total de plus de 7000 cystectomies [6].

Actuellement, à travers des études prospectives randomisées, le programme RAC appliqué à la cystectomie montre des avantages non négligeables avec une durée de séjour plus courte et une réduction des complications postopératoires sans modifier les résultats oncologiques et fonctionnels $[7,8,9]$.

\section{Les paramètres peropératoires}

Pour les paramètres préopératoires du programme ERAS, plus de la moitié des urologues maghrébins suivent plusieurs recommandations : l'arrêt du tabac 8 semaines avant le geste opératoire, l'évaluation de l'état nutritionnel et le temps de jeune de 2 à $4 \mathrm{~h}$.

Les complications respiratoires post-cystectomie surviennent dans 2 à $8 \%$ des cas, ce qui rend l'optimisation des maladies respiratoires en préopératoire très légitimes [10]. D'après la méta-analyse de Katsura [11], la réduction postopératoire de risque d'atélectasie et de pneumopathie était statistiquement significative s'il y'a une réduction de risque (RR) d'atélectasie de 0.53 ( $R R=0,53$, IC à 95\% 0,34-0,82) et de pneumopathie de 0.45 ( $R R=0,45$, IC 95\% 0,26-0,77). Il est important de réduire le risque de toutes les pathologies respiratoires et de proposer une aide pour le sevrage tabagique [12].

Un arrêt de la consommation du tabac entre 4 à 8 semaines avant le geste a montré une diminution des complications pour la chirurgie colorectale [3]. Le sevrage tabagique trop près de la chirurgie pourrait être un facteur de risque d'iléus post-opératoire, il faut penser à la substitution nicotinique [13].

L'évaluation de l'état nutritionnel du patient est indispensable en préopératoire car c'est un facteur de mortalité de la cystectomie [14]. Plusieurs sociétés savantes à savoir la SFAR (Société Française d'Anesthésie et de Réanimation) et la SFNEP (Société Francophone Nutrition Clinique et Métabolique) recommandent que tous les patients qui vont subir une cystectomie devront bénéficier d'une évaluation nutritionnelle qui comporte : le poids actuel (IMC), la perte de poids, la mesure de l'albuminémie, le « nutritional risk score » $[15,16,17]$.

Les urologues maghrébins gardent toujours quelques anciennes pratiques dans la préparation digestive avant la cystectomie, comme l'utilisation des produits hyperosmolaires. La préparation colique a fait l'objet de plusieurs travaux en chirurgie colorectale. Quelques travaux montrent qu'elle pourrait exercer un effet bénéfique sur le délai de reprise du transit intestinal $[18,19]$. Cependant, deux essais randomisés contrôlés, dont un avec adjonction d'une antibiothérapie orale par Erythromycine et Métronidazole, ne retrouvent pas d'intérêt de la préparation digestive sur le délai de reprise du transit intestinal après cystectomie. Pour les lavements prérectaux en préopératoire, aucune étude dans la littérature n'a montré un intérêt sur la reprise du transit. La préparation digestive avec un régime sans résidu peut entrainer des dégâts délétères sur l'état nutritionnel du patient et le processus de cicatrisation. Il est indiqué en cas ou le patient présente un iléus en préopératoire par exemple, les blessés médullaires, la constipation chronique [20,21].

Pour la période de jeûne, les recommandations de l'ERAS stipulent une durée de 6 heures pour l'alimentation solide 
et entre 2 à 4 heures pour les liquides et les solutions glucidiques [22]. Ceci ne concerne pas les patients diabétiques insulinodépendants, les neuropathies et les patients qui ont un obstacle gastrique (occlusion intestinale) [23].

\section{Les paramètres peropératoires}

Les équipes maghrébines préviennent l'hypothermie par tous les moyens disponibles. Cependant, ils privilégient l'abord classique que la voie coelioscopique qui représente $3.2 \%$ dans la pratique de la cystectomie. La majorité des urologues interrogés déclarent que leurs équipes d'anesthésie pratiquent l'anesthésie générale combinée à l'analgésie péridurale. L'analgésie péridurale thoracique représente le gold standard dans la gestion de la douleur en postopératoire. Elle a montré son avantage à la PCA Morphine dans la réduction de la quantité des opiacés en post-opératoire $[24,25,26]$.

La voie d'abord laparoscopique pour la cystectomie répond au principe de la RAAC. Elle minimise les traumatismes induits et la réponse inflammatoire. Les données de la littérature sont très mitigées, la voie classique reste le standard pour la plupart des sociétés savantes. Les données de la littérature donnent quelques avantages à la voie mini-invasive coelioscopique ou aidée au robot parce qu'elle diminue d'abord les complications postopératoires, les pertes sanguines et la durée du séjour $[27,28]$. Le surcoût net de la procédure laparoscopique robot-assistée rend cette technique inaccessible par tout le monde [29,30].

Le drainage du site opératoire est très discuté entre les auteurs sachant qu'il reste une recommandation en fonction des protocoles et les habitudes du service. Selon la conférence de consensus des experts de l'EAU 2016, 61\% des experts recommandent de ne pas pratiquer le drainage que chez des patients bien sélectionnés [31]. 82\% des experts proposent un drainage non aspiratif de charrière 21 du site opératoire. L'ablation du drain se fait à partir de J1 s'il n'y avait pas de doute sur la survenue d'une fistule urinaire. Actuellement, le drainage est recommandé sans préciser ni le type, ni la durée.

On a noté que la grande majorité des urologues ne sont pas au courant de la gestion des fluides en peropératoire en croyant que le patient ne doit pas être en hypovolémie seulement. Il est reconnu qu'un remplissage excessif est tout autant délétère qu'un remplissage insuffisant responsable des complications postopératoires. Le remplissage excessif est responsable de lésion de l'endothélium vasculaire, iléus postopératoire, la fistule digestive, les complications cardiorespiratoires postopératoires et l'augmentation de la durée de séjour [32].

\section{Les paramètres postopératoires}

On note une adhésion forte des urologues maghrébins au programme fast track avec un taux de $75.6 \%$ pour la non utilisation des morphiniques. La mobilisation précoce des patients après chirurgie lourde est très largement recommandée dans la littérature grâce aux bénéfices engendrés sur la fonction cardiorespiratoire, psychologique et la diminution de la durée de séjour [33]. Dans les protocoles, il est proposé de commencer à J0 par une mise au bord du lit, puis à J1 une mobilisation hors du lit avec lever et mise au fauteuil. A J2, on effectue quelques pas de marche dans la chambre pour notamment se rendre dans la salle de bain [34].

La gestion des nausées et vomissements en postopératoire est mal prise en charge par les urologues maghrébins avec une prescription des antiémétiques estimée à 34.4\%. Cependant, la gestion des nausées et vomissements figure dans les recommandations de la réhabilitation post-cystectomie. Les facteurs de risque de NVPO ont été clairement identifiés et constituent actuellement un score validé (score d'Apfel) [35].

Les principaux facteurs de risques sont : le sexe féminin, le " non tabagisme ", les antécédents de mal des transports et la prescription d'opiacés postopératoires. Il est à noter qu'il existe une relation linéaire entre la consommation de morphiniques postopératoires et l'incidence de nausées et vomissements [36]. La prophylaxie antiémétique médicamenteuse recommandée dans la littérature est constituée de l'ondansétron, le dropéridol à faible dose (inférieure ou égale à 1,25 mg) et la dexaméthazone ; chacun de ces 3 produits faisant baisser de $50 \%$ le risque de nausées et vomissements [37].

Concernant l'alimentation postopératoire, 52.3\% d'urologues estiment que l'alimentation parentérale doit être proscrite en postopératoire. Les principales règles de cette alimentation reposent sur la réalimentation précoce et la nutrition orale [15]. La nutrition parentérale à courte durée (moins de 7 jours) ne doit pas être systématique chez tous les patients vu le risque accru de complication de cette voie [38]. Le jeune court avec l'alimentation orale précoce, diminue les complications postopératoires, accélère le transit et écourte la durée de séjour [39].

Cette enquête déclarative concernant l'adhésion des urologues maghrébins aux recommandations de la réhabilitation améliorée post-cystectomie représente la 1ère étude à notre connaissance qui décrit les pratiques réelles dans le management de la cystectomie radicale. C'est une étude qui a touché les urologues marocains, algériens et tunisiens qui ont participé aux congrès de leurs sociétés savantes de l'année 2017.

L'auto-questionnaire a été soumis aux médecins pendant les séances plénières de chaque congrès. Cette méthode a été choisie afin d'élargir l'échantillon. Ceci a permis d'analyser 
plus de $57,7 \%$ de questionnaires (383 questionnaires soumis, 221 retournés et analysés). Ce taux de réponse est un point fort de l'échantillonnage, bien que la soumission de l'auto-questionnaire aux urologues présents lors des congrès, donne des résultats non exhaustifs des pratiques. Ceci n'empêche pas de généraliser ces résultats sur l'ensemble des urologues maghrébins car la population de l'étude est représentée par plus de $43 \%$ de médecins hospitalo-universitaires qui pratiquent le plus de cystectomies dans leur activité quotidienne. L'étude s'intéresse à un échantillon d'urologues actifs qui participent aux congrès et qui essaient de mettre à jour leurs connaissances.

Le reste des médecins qui n'ont pas souhaité répondre au questionnaire sont des urologues qui ne pratiquent pas souvent ce genre de techniques chirurgicales et à défaut, d'un recrutement très faible.

Il faut noter qu'au Maghreb il n'existe pas de programme de réhabilitation améliorée post- cystectomie établie par les sociétés savantes locales. Depuis des années, plusieurs programmes européens de réhabilitation ont été élaborés dans la prise en charge du cancer colorectal. La chirurgie colorectale est probablement la procédure qui a fait l'objet de plus grand nombre de publications dans ce domaine. Toutes les méta-analyses montrent que l'application de ces programmes réduit la durée de séjour et le taux de complications [40]. En France, il y'a eu une évolution règlementaire et législative récente qui favorisé le développement et la création d'un dispositif global, coordonnée et illustrée par le guide de l'HAS « sécurité du patient ». Dans ce sens, des sociétés savantes chirurgicales, anesthésiques et nutritionnelles ont dépêché leurs experts pour établir des programmes conformes à cette législation et créer le groupe francophone interdisciplinaire de réhabilitation après chirurgie (GRACE) au début de l'année 2014. C'est une association pluridisciplinaire ayant pour but de favoriser le développement et la diffusion de la réhabilitation améliorée après chirurgie. Il concerne toutes les spécialités chirurgicales pouvant bénéficier de la réhabilitation améliorée, notamment la chirurgie digestive, hépato-bilio-pancréatique, la chirurgie cardiovasculaire, thoracique, la gynécologie, l'orthopédie et l'urologie [41,42].

Dans la littérature, il n'existe pas de questionnaire spécifique valide pour évaluer l'adhésion des urologues au programme de RAC. Le questionnaire a été établie en fonction des recommandations du programme d'ERAS de 2013 par une équipe pluridisciplinaire qui prend en charge les cystectomies dans notre service.

La majorité des urologues maghrébins suivent plusieurs recommandations du programme ERAS comme : l'arrêt du tabac 8 semaines avant le geste opératoire, l'évaluation de l'état nutritionnel. Leurs équipes d'anesthésie préviennent l'hypothermie et pratiquent l'anesthésie générale combinée à l'analgésie péridurale. Le programme fast track reste très adopté en postopératoire. Il reste quelques pratiques très répandues qui ne sont plus recommandées par ERAS comme l'utilisation des produits hyperosmolaires dans la préparation digestive, l'abord classique dans la cystectomie et la non prescription des antiémétiques. Dans l'ensemble, il existe un suivi moyen des paramètres préopératoires, peropératoires et postopératoires.

Selon les résultats de l'étude, l'état nutritionnel en préopératoire et la préparation digestive avec un régime sans résidu diminue la durée d'hospitalisation. Cette conclusion a été approuvée par des études de haut niveau de preuve. En effet les autres paramètres du programme ERAS ont prouvé leurs efficacités sur la diminution de la morbidité opératoire et le séjour hospitalier.

L'enquête a permis de mettre le point sur les pratiques actuelles des urologues maghrébins et leurs équipes (anesthésiste, infirmier, kinésithérapeute...) dans la prise en charge du perioprératoire de la cystectomie radicale. Elle permet de voir à quel point les pratiques maghrébines rejoignent les recommandations internationales afin d'agir et de sensibiliser la communauté d'urologie sur la nécessité de la réhabilitation améliorée après chirurgie. En effet, vu le bon suivi des items d'ERAS, la création et l'installation d'un programme de réhabilitation maghrébin certifié par les sociétés savantes demeure une perspective très encourageante pour améliorer la prise en charge des malades et réduire le coût économique.

\section{Conclusion}

Cette enquête montre que plus de la moitié des urologues maghrébins déclare qu'ils suivent les recommandations du programme de réhabilitation améliorée afin de diminuer la morbidité et de réduire la durée d'hospitalisation. L'autorisation de l'alimentation postopératoire précoce reste très peu suivie. Etablir un programme de réhabilitation commun à tous les pays du Maghreb devient une nécessité afin de codifier la prise en charge des cystectomies et réduire la comorbidité peropératoire.

\section{Conflits d'intérêt}

Les auteurs déclarent n'avoir aucun conflit d'intérêt. 


\section{Références bibliographiques}

[1]: Kehlet H. Multimodal approach to control postoperative pathophysiology andrehabilitation. British Journal of Anaesthesia 1997 May;78(5):606e17.

[2] : HAS. Programmes de récupération améliorée après chirurgie (RAAC) : état des lieux etperspectives. 2016.

[3]: Cerantola $Y$, Valerio M, Persson B, Jichlinski P, Ljungqvist 0 , Hubner $M$, et al. Guidelines for perioperative care after radical cystectomy for bladder cancer: Enhanced Recovery After Surgery $(\operatorname{ERAS}((R)))$ society recommendations. Clinical nutrition. 2013 Dec;32(6):879-87. PubMed PMID: 24189391.

[4]: Varadhan KK, Neal KR, Dejong CH, Fearon KC, Ljungqvist O, Lobo DN. Theenhanced recovery after surgery (ERAS) pathway for patients undergoingmajor elective open colorectal surgery: a meta-analysis of randomizedcontrolled trials. Clinical Nutrition (Edinburgh, Scotland) 2010 Aug;29(4):434e40.

[5]: Shabsigh A, Korets R, Vora KC, Brooks CM, Cronin AM, Savage $C$, et al.Defining early morbidity of radical cystectomy for patients with bladdercancer using a standardized reporting methodology. European Urology 2009Jan;55(1):164e74.

[6]: World Health Organization (WHO) Consensus Conference on Bladder Cancer,Hautmann RE, Abol-Enein H, Hafez K, Haro I, Mansson W, et al. Urinary diversion.Urology. 2007 Jan;69(1 Suppl):17-49.

[7] : Karl H.PangRuthGroves cSuresh Venugopal dAidan P.Noon bJames W.F.Cattoab .Prospective Implementation of Enhanced Recovery After Surgery Protocols to Radical Cystectomy. European UrologyVolume 73, Issue 3, March 2018, Pages 363-371.

[8]:Mark D.Tyson, Sam S.Chang. Enhanced Recovery Pathways Versus Standard Care After Cystectomy: A Meta-analysis of the Effect on Perioperative Outcomes. European Urology.Volume 70, Issue 6, December 2016, Pages 995-1003.

[9]: Raed A.AzharBernardBochnerJamesCattoAlvin C.GohJohnKellyHiten D.PatelRaj S.PruthiGeorge N.ThalmannMihirDesai. Enhanced Recovery after Urological Surgery: A Contemporary Systematic Review of Outcomes, Key Elements, and Research Needs. European UrologyVolume 70, Issue 1, July 2016, Pages 176-187.

[10]: Froehner M, Brausi MA, Herr HW, Muto G, Studer UE. Complications following radical cystectomy for bladder cancer in the elderly. Eur Urol. 2009 Sep;56(3):443-54. PubMed PMID: 19481861.

[11]:Katsura M, Kuriyama A, Takeshima T, Fukuhara S, Furukawa TA. Preoperative inspiratory muscle training for postoperative pulmonary complications in adults undergoing cardiac and major abdominal surgery. The Cochrane database of systematic reviews. 2015 (10):CD010356. PubMed PMID: 26436600.

[12]:Nygren J, Thacker J, Carli F, Fearon KC, Norderval S, Lobo DN, et al. Guidelines for perioperative care in elective rectal/pelvic surgery: Enhanced Recovery After Surgery (ERAS(R)) Society recommendations. Clinical nutrition. 2012 Dec;31(6):801-16. PubMed PMID: 23062720 .

[13]: Hollenbeck BK, Miller DC, Taub D, Dunn RL, Khuri SF, Henderson
WG, et al. Identifying risk factors for potentially avoidable complications following radical cystectomy. J Urol. 2005 Oct;174(4 Pt 1):1231-7; discussion 7. PubMed PMID: 16145376.

[14]: Gregg JR, Cookson MS, Phillips S, Salem S, Chang SS, Clark PE, et al. Effect of preoperative nutritional deficiency on mortality after radical cystectomy for bladder cancer. J Urol. 2011 Jan;185(1):90-6. PubMed PMID: 21074802. Pubmed Central PMCID: 3049248.

[15] : Chambrier C, Sztark F, Societe Francophone de nutrition clinique et $\mathrm{m}$, Societe francaise d'anesthesie et $\mathrm{r}$. French clinical guidelines on perioperative nutrition. Update of the 1994 consensus conference on perioperative artificial nutrition for elective surgery in adults. Journal of visceral surgery. 2012 Oct;149(5):e325-36. PubMed PMID: 23107793.

[16]: Gibbs J, Cull W, Henderson W, Daley J, Hur K, Khuri SF. Preoperative serum albumin level as a predictor of operative mortality and morbidity: results from the National VA Surgical Risk Study. Arch Surg. 1999 Jan;134(1):36-42. PubMed PMID: 9927128.

[17]: Kondrup J, Rasmussen HH, Hamberg O, Stanga Z, Ad Hoc EWG. Nutritional risk screening (NRS 2002): a new method based on an analysis of controlled clinical trials. Clinical nutrition. 2003 Jun;22(3):321-36. PubMed PMID: 12765673.

[18]: Kiran RP, Murray AC, Chiuzan C, Estrada D, Forde K. Combined preoperative mechanical bowel preparation with oral antibiotics significantly reduces surgical site infection, anastomotic leak, and ileus after colorectal surgery. Ann Surg. 2015 Sep;262(3):416-25; discussion 23-5. PubMed PMID: 26258310.

[19]: Park HK, Kwak C, Byun SS, Lee E, Lee SE. Early removal of nasogastric tube after cystectomy with urinary diversion: does postoperative ileus risk increase? Urology. 2005 May;65(5):905-8. PubMed PMID: 15882721.

[20]: Xu R, Zhao X, Zhong Z, Zhang L. No advantage is gained by preoperative bowel preparation in radical cystectomy and ileal conduit: a randomized controlled trial of 86 patients. International urology and nephrology. 2010 Dec;42(4):947-50. PubMed PMID: 20390354.

[21]: Hashad MM, Atta M, Elabbady A, Elfiky S, Khattab A, Kotb A. Safety of no bowel preparation before ileal urinary diversion. BJU Int. 2012 Dec;110(11 Pt C):E1109-13. PubMed PMID: 23167296.

[22]: Smith I, Kranke P, Murat I, Smith A, O'Sullivan G, Soreide E, et al. Perioperative fasting in adults and children: guidelines from the European Society of Anaesthesiology. European journal of anaesthesiology. 2011 Aug;28(8):556-69. PubMed PMID: 21712716.

[23]: Definition, incidence, risk factors, and prevention of paralytic ileus following radical cystectomy: a systematic review, Ramirez JA, 2013, Eur Urol).

[24]: Werawatganon T, Charuluxanun S. Patient controlled intravenous opioid analgesia versus continuous epidural analgesia for pain after intra-abdominal surgery. The Cochrane database of systematic reviews. 2005 (1):CD004088. PubMed PMID: 15674928.

[25] : Wu CL, Cohen SR, Richman JM, Rowlingson AJ, Courpas GE, Cheung $\mathrm{K}$, et al. Efficacy of postoperative patient-controlled and 
continuous infusion epidural analgesia versus intravenous patient-controlled analgesia with opioids: a meta-analysis. Anesthesiology. 2005 Nov;103(5):1079-88; quiz 109-10. PubMed PMID: 16249683.

[26]: Rigg JR, Jamrozik K, Myles PS, Silbert BS, Peyton PJ, Parsons RW, et al. Epidural anaesthesia and analgesia and outcome of major surgery: a randomised trial. Lancet. 2002 Apr 13;359(9314):1276-82. PubMed PMID: 11965272.

[27]: Novara G, Catto JW, Wilson T, Annerstedt M, Chan K, Murphy DG, et al. Systematic review and cumulative analysis of perioperative outcomes and complications after robot-assisted radical cystectomy. Eur Urol. 2015 Mar;67(3):376-401. PubMed PMID: 25560798.

[28] : Li K, Lin T, Fan X, Xu K, Bi L, Duan Y, et al. Systematic review and meta-analysis of comparative studies reporting early outcomes after robot-assisted radical cystectomy versus open radical cystectomy. Cancer treatment reviews. 2013 Oct;39(6):551-60. PubMed PMID: 23273846.

[29]: Hu JC, Chughtai B, O'Malley P, Halpern JA, Mao J, Scherr DS, et al. Perioperative Outcomes, Health Care Costs, and Survival After Robotic-assisted Versus Open Radical Cystectomy: A National Comparative Effectiveness Study. Eur Urol. 2016 Apr 28. PubMed PMID: 27133087.

[30]: Yu HY, Hevelone ND, Lipsitz SR, Kowalczyk KJ, Nguyen PL, Choueiri TK, et al. Comparative analysis of outcomes and costs following open radical cystectomy versus robot-assisted laparoscopic radical cystectomy: results from the US Nationwide Inpatient Sample. Eur Urol. 2012 Jun;61(6):1239-44. PubMed PMID: 22482778.

[31]: Collins JW, Patel H, Adding C, Annerstedt M, Dasgupta P, Khan SM, et al. Enhanced Recovery After Robot-assisted Radical Cystectomy: EAU Robotic Urology Section Scientific Working Group Consensus View. Eur Urol. 2016 May 24. PubMed PMID: 27234997.

[32]: Brandstrup B, Tonnesen H, Beier-Holgersen R, Hjortso E, Ording $\mathrm{H}$, Lindorff-Larsen $\mathrm{K}$, et al. Effects of intravenous fluid restriction on postoperative complications: comparison of two perioperative fluid regimens: a randomized assessor-blinded multicenter trial. Ann Surg. 2003 Nov;238(5):641-8. PubMed PMID: 14578723. Pubmed Central PMCID: 1356139.

[33]: Drolet A, DeJuilio P, Harkless S, Henricks S, Kamin E, Leddy EA, et al. Move to improve: the feasibility of using an early mobility protocol to increase ambulation in the intensive and intermediate care settings. Physical therapy. 2013 Feb;93(2):197-207. PubMed PMID: 22976447.

[34]: Koupparis A, Villeda-Sandoval C, Weale N, El-Mahdy M, Gillatt $D$, Rowe E. Robot-assisted radical cystectomy with intracorporeal urinary diversion: impact on an established enhanced recovery protocol. BJU international. 2015 Dec;116(6):924-31. PubMed PMID: 25943158.

[35]: Apfel CC, Heidrich FM, Jukar-Rao S, Jalota L, Hornuss C, Whelan RP, et al. Evidence-based analysis of risk factors for postoperative nausea and vomiting. $\mathrm{Br} J$ Anaesth. $2012 \mathrm{Nov}$;109(5):742-53. PubMed PMID: 23035051
[36]: Roberts GW, Bekker TB, Carlsen HH, Moffatt CH, Slattery PJ, McClure AF. Postoperative nausea and vomiting are strongly influenced by postoperative opioid use in a dose-related manner. Anesth Analg. 2005 Nov;101(5):1343-8. PubMed PMID: 16243992.

[37]: Gan TJ, Jiao KR, Zenn M, Georgiade G. A randomized controlled comparison of electro-acupoint stimulation or ondansetron versus placebo for the prevention of postoperative nausea and vomiting. Anesth Analg. 2004 Oct;99(4):1070-5, table of contents. PubMed PMID: 15385352.

[38]: Klein S, Kinney J, Jeejeebhoy K, Alpers D, Hellerstein M, Murray $M$, et al. Nutrition support in clinical practice: review of published data and recommendations for future research directions. Clinical nutrition. 1997 Aug;16(4):193-218. PubMed PMID: 16844599.

[39]: Andersen HK, Lewis SJ, Thomas S. Early enteral nutrition within $24 \mathrm{~h}$ of colorectal surgery versus later commencement of feeding for postoperative complications. The Cochrane database of systematic reviews. 2006 (4):CD004080. PubMed PMID: 17054196.

[40]: Gustafsson UO, Scott MJ, Schwenk W, Demartines N, Roulin D, Francis N, et al. Guidelines for perioperative care in elective colonic surgery: Enhanced Recovery After Surgery (ERAS(R)) Society recommendations. Clinical nutrition. 2012 Dec;31(6):783-800. PubMed PMID: 23099039.

[41] HAS. «La sécurité des patients ", Mettre en œuvre la gestion des risques associés aux soins en établissement de santé. Mars 2012.

[42] : Le Groupe francophone interdisciplinaire de réhabilitation améliorée en chirurgie (GRACE) https://www.grace-asso.fr. 
